\title{
Moisture compatible and recyclable indium (III) chloride catalyzed and microwave assisted efficient route to substituted $1 H$-quinolin-2- ones
}

\author{
I. R. Siddiqui,* Shayna Shamim, Archana Singh, Vishal Srivastava, and Sanjay Yadav \\ Laboratory of Green Synthesis, Department of Chemistry \\ University of Allahabad, Allahabad-211002, India \\ Email: irsiddiquiau@ rediffmail.com
}

DOI: $\underline{\text { http://dx.doi.org/10.3998/ark.5550190.0011.b19 }}$

\begin{abstract}
An efficient three component synthesis of highly functionalized 4-methyl-1H-quinolin-2-ones in one-pot from readily available coumarin, hydrazine and isatin catalyzed by a recyclable and moisture compatible $\mathrm{InCl}_{3}$ under microwave irradiation has been developed. The synthesis involves a $\mathrm{InCl}_{3}$ catalyzed dehydrative nucleophilic substitution on the lactone moiety of coumarin by the isatin hydrazone resulting in the formation of $\mathrm{N}$-substituted lactams, 6substituted-4-methyl-1-(2-oxo-1,2-dihydroindol-3-ylidenamino)-1H-quinolin-2-ones. The coumarin based transformation into substituted $1 H$-quinolin-2-ones proceeded smoothly with quantitative yields at ambient temperature.
\end{abstract}

Keywords: Quinolin-2-one, one-pot, microwave irradiation, indium (III) chloride

\section{Introduction}

The presence of a quinoline nucleus in the frame work of various pharmacologically active compounds with antiasthmatic, ${ }^{1}$ antibacterial, ${ }^{2}$ antifungal, ${ }^{3}$ antimalarial, ${ }^{4}$ antiviral, ${ }^{5}$ antiinflammatory ${ }^{6}$ activities continue to promote their synthetic efforts. In addition, quinolines are valuable synthons used for the preparation of nano- and meso structures with enhanced electronic and photonic properties. ${ }^{7}$ A series of compounds derived from 8-hydroxyquinolines and styryl quinoline were recently synthesized as potential HIV-1 integrase inhibitors., ${ }^{8,9}$ Similarly, isatin $\beta$-thiosemicarbazone derivatives were found to demonstrate a range of antiviral activities against Maloney leukemia virus, vaccinia virus ${ }^{10-13}$ and inhibit HIV-1 replication. ${ }^{14}$ Furthermore, isatin derivatives were also found to show antiviral activity against HIV-2 and HIV-3 in MT-4 cells. ${ }^{15}$ Because of these biodynamic properties associated with quinolin-2-one and isatin, quinolin-2-ones incorporating an isatin moiety appear to be attractive scaffolds to provide 
a chemical diverse drug like library. The classical synthetic protocols for quinolin-2-one derivatives which suffer from disadvantages such as low yields, lack of easy availability/preparation of reagents, prolonged reaction time, multistep procedures, harsh reaction conditions etc. has increased our interest in making an effort for the development of environmentally benign and more atom economical simple protocols for the synthesis of $1 H$ quinolin-2-one derivatives.

The development and applications of catalytic reactions is nowadays worldwide documented and discussed. In recent years there has been a phenomenal increase in the use of catalysts in synthesis of pharmacologically important organic compounds especially heterocycles. Recently, indium (III) chloride has emerged as a powerful Lewis acid catalyst imparting high chemo- and regioselectivity in various transformations. ${ }^{16}$ The versatility of indium (III) chloride because of its non-toxic nature, recyclability, readily availability, high selectivity ${ }^{17}$ and moisture compatibility $^{18}$ encouraged us to carry out a coumarin based transformation into $1 H$-quinolin-2ones in the presence of hydrazine under benign reaction conditions.

Multi component reactions (MCRs) are powerful synthetic tools which have changed the landscape of organic and medicinal chemistry due to their environment friendliness, atom economy and their ability to generate large library of compounds. ${ }^{19}$

Recent years have also witnessed a phenomenal growth in the application of microwave activation in organic synthesis. ${ }^{20}$ The application of microwave activation in conjugation with metal halide catalysts in one-pot multi-component reactions provides an environmentally benign process associated with higher yield of products, mild reaction conditions, a significant reduction in reaction time, an enhanced reaction rate, all of which are additional eco-friendly attributes in the context of green chemistry. ${ }^{21}$

As part of our program to develop new, simple, efficient, clean, selective, environmentally benign methodologies for the synthesis of potential bioactive heterocycles we report here the catalytic activity of $\mathrm{InCl}_{3}$ in a one-pot three-component efficient and clean synthesis of $1 \mathrm{H}$ quinolin-2-one from coumarin in the presence of hydrazine ${ }^{22}$ (Scheme 1).

\section{Result and Discussion}

We have reported here a novel one-pot strategy for the synthesis of, hitherto, unknown highly substituted $1 H$-quinolin-2-one derivatives based on the MCR of isatin, hydrazine, and coumarins under microwave irradiation in the presence of $10 \mathrm{~mol} \%$ of $\mathrm{InCl}_{3}$ using ethanol as a solvent (Scheme 1). The usual work-up gave the corresponding $1 H$-quinolin-2-ones in excellent yields. 


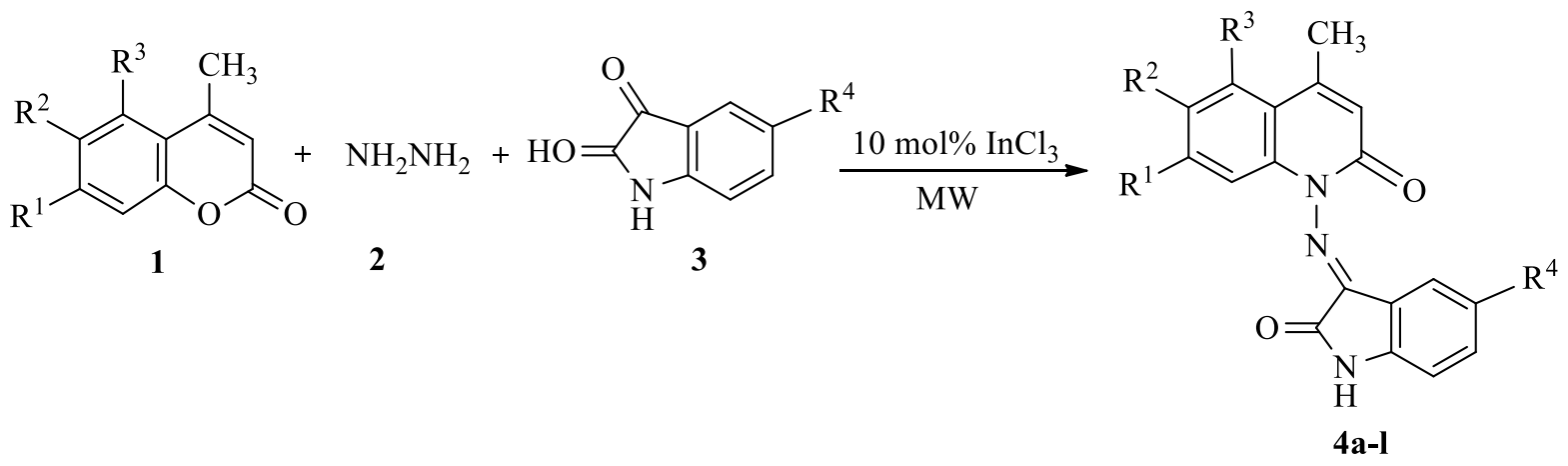

Scheme 1. $\mathrm{InCl}_{3}$ catalyzed microwave assisted one-pot synthesis of quinolinones.

\begin{tabular}{cllll}
\hline Compound & $\mathrm{R}^{1}$ & $\mathrm{R}^{2}$ & $\mathrm{R}^{3}$ & $\mathrm{R}^{4}$ \\
\hline $\mathbf{4 a}$ & $\mathrm{OH}$ & $\mathrm{H}$ & $\mathrm{H}$ & $\mathrm{H}$ \\
$\mathbf{4 b}$ & $\mathrm{H}$ & $\mathrm{OH}$ & $\mathrm{H}$ & $\mathrm{H}$ \\
$\mathbf{4} \mathbf{c}$ & $\mathrm{OH}$ & $\mathrm{H}$ & $\mathrm{OH}$ & $\mathrm{H}$ \\
$\mathbf{4 d}$ & $\mathrm{OCH}$ & $\mathrm{H}$ & $\mathrm{H}$ & $\mathrm{H}$ \\
$\mathbf{4} \mathbf{e}$ & $\mathrm{OH}$ & $\mathrm{H}$ & $\mathrm{H}$ & $\mathrm{NO}_{2}$ \\
$\mathbf{4}$ & $\mathrm{H}$ & $\mathrm{OH}$ & $\mathrm{H}$ & $\mathrm{NO}_{2}$ \\
$\mathbf{4}$ & $\mathrm{OH}$ & $\mathrm{H}$ & $\mathrm{OH}$ & $\mathrm{NO}_{2}$ \\
$\mathbf{4}$ & $\mathrm{OCH}$ & $\mathrm{H}$ & $\mathrm{H}$ & $\mathrm{NO}_{2}$ \\
$\mathbf{4}$ & $\mathrm{OH}$ & $\mathrm{H}$ & $\mathrm{H}$ & $\mathrm{CH}_{3}$ \\
$\mathbf{4}$ & $\mathrm{H}$ & $\mathrm{OH}$ & $\mathrm{H}$ & $\mathrm{CH}_{3}$ \\
$\mathbf{4}$ & $\mathrm{OH}$ & $\mathrm{H}$ & $\mathrm{OH}$ & $\mathrm{CH}_{3}$ \\
$\mathbf{4}$ & $\mathrm{OCH}$ & $\mathrm{H}$ & $\mathrm{H}$ & $\mathrm{CH}_{3}$ \\
\hline
\end{tabular}

To generalize this reaction we have reacted various substituted isatin and coumarin derivatives and isolated the corresponding $1 H$-quinolin-2-ones in $80-90 \%$ yield (Table 1 ). In all cases the reaction proceeded smoothly in the presence of $\mathrm{InCl}_{3}$. When $\mathrm{InCl}_{3}$ was recycled and reused the yield of the products were almost same indicating that there is no drop in activity of the $\mathrm{InCl}_{3}$. The results obtained from $\mathrm{InCl}_{3}$ catalyzed microwave assisted synthesis of substituted $1 H$-quinolin-2-one derivatives in a one-pot process are summarized in Table 1. The reaction proceeded quantitatively in ethanol. When other solvents like THF, acetic acid were employed or when no solvent was used result obtained were disappointing and reactant itself was recovered. $\mathrm{InCl}_{3}$ gave better yields as compared to other Lewis acid, such as $\mathrm{SnCl}_{4}, \mathrm{FeCl}_{3}$, and $\mathrm{CuCl}_{2}$. This was studied by varying four Lewis acids and kept all the condition same as mentioned in experiment section. The yields obtained in four different experiments were shown in Table 2. 
Table 1. $\mathrm{InCl}_{3}$ catalyzed microwave assisted one-pot three component synthesis of quinolinones 4a-l

\begin{tabular}{|c|c|c|c|c|c|}
\hline \multirow[t]{3}{*}{ Compound } & \multirow{3}{*}{$\begin{array}{c}\text { Time } \\
\text { MW (min). }\end{array}$} & \multicolumn{3}{|c|}{ Yield (\%) } & \multirow[t]{3}{*}{ m.p. ${ }^{\circ} \mathrm{C}$} \\
\hline & & \multicolumn{2}{|c|}{ with $\mathrm{InCl}_{3}$} & \multirow[t]{2}{*}{ without catalyst } & \\
\hline & & $\mathrm{a}$ & $\mathrm{b}$ & & \\
\hline $4 \mathbf{a}$ & 5 & 87 & 86 & 42 & $156-158$ \\
\hline $4 b$ & 5 & 83 & 83 & 46 & $157-159$ \\
\hline $4 c$ & 4 & 86 & 86 & 50 & $164-166$ \\
\hline $4 d$ & 4 & 83 & 83 & 45 & $145-147$ \\
\hline $4 e$ & 5 & 85 & 84 & 48 & $168-169$ \\
\hline $4 f$ & 6 & 81 & 81 & 43 & $167-169$ \\
\hline $4 g$ & 5 & 84 & 84 & 47 & $172-173$ \\
\hline $4 h$ & 6 & 81 & 81 & 41 & $165-166$ \\
\hline $4 \mathbf{i}$ & 6 & 82 & 82 & 44 & $162-164$ \\
\hline $4 \mathbf{j}$ & 5 & 84 & 84 & 43 & $163-164$ \\
\hline $4 \mathbf{k}$ & 6 & 91 & 90 & 41 & $170-172$ \\
\hline 41 & 5 & 89 & 89 & 49 & $152-153$ \\
\hline
\end{tabular}

a: Isolated yield with $\mathrm{InCl}_{3}$ catalyst used $1^{\text {st }}$ time

b: Isolated yield with $\mathrm{InCl}_{3}$ catalyst used $2^{\text {nd }}$ time

This supports that $\mathrm{InCl}_{3}$ is much better Lewis acid for this reaction. The synthesis of highly functionalized 4-methyl-1H-quinolin-2-ones in a one-pot reaction has been developed and it was found that the envisaged three-component synthesis (Scheme 1) was successful. Experiments were completed within 4-6 min. as monitored by TLC showing the disappearance of the starting materials.

Table 2. Effect of different Lewis acids on yield (\%)

\begin{tabular}{ccc}
\hline Entry & Lewis acid & Yields \% \\
\hline 1 & $\mathrm{InCl}_{3}$ & 87 \\
2 & $\mathrm{CuCl}_{2}$ & 46 \\
3 & $\mathrm{FeCl}_{3}$ & 41 \\
4 & $\mathrm{SnCl}_{4}$ & 35 \\
\hline
\end{tabular}

To ascertain whether the $\mathrm{MW}$ and $\mathrm{InCl}_{3}$ combination truly improved the yield or simply increased conversion rates, we performed a comparative experiment in the absence of the catalyst $\mathrm{InCl}_{3}$. It was found that the products were obtained only in $40-50 \%$ yield, confirming the functional role of $\mathrm{InCl}_{3}$ as the catalyst. The rate enhancement can be rationalized on the basis of the formation of a dipolar activated complex II in these reactions (Scheme 2) and the greater stabilization of the dipolar activated complex by dipole-dipole interactions with the 
electromagnetic field of the microwave may reduce the activation energy $\left(\mathrm{G}^{*}\right)$ resulting in the rate enhancement.<smiles>[R1]c1ccc2c(c1)C(=O)C(=O)N2</smiles><smiles>[R]c1ccc2c(c1)/C(=N/NC1([O-])C=C(C)c3c(cc([R])c([R])c3[R])O1)C(=O)NC2=NN</smiles>

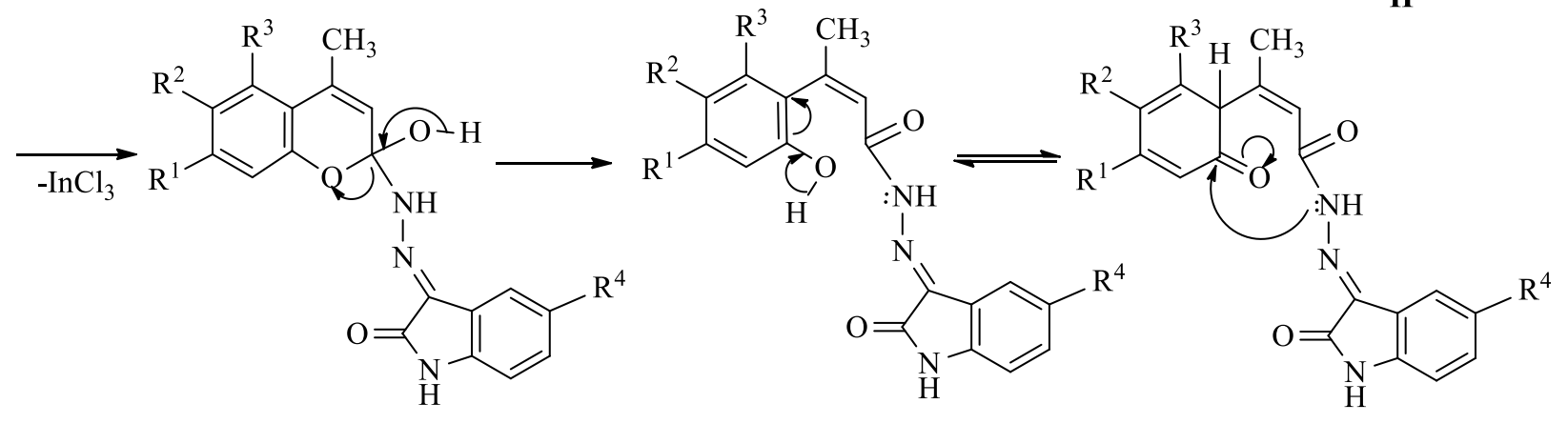

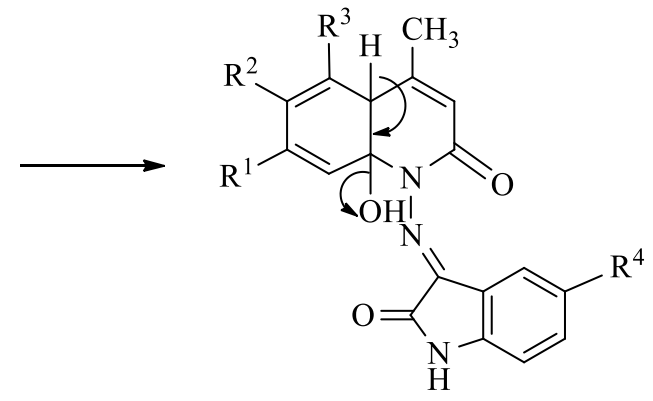<smiles>[R]c1ccc2c(c1)/C(=N/n1c3c(C)cc([R])c-3c([R])c([R])cc1=O)NC2=O</smiles>

Scheme 2. Proposed mechanism for the synthesis of quinolin-2-ones in the presence of $\mathrm{InCl}_{3}$ as catalyst.

Spectral analysis of 4a-I supported the success of the MW-mediated one-pot triple condensation. The ${ }^{1} \mathrm{H}$ NMR spectra of 4 a-l exhibited multiplets in the region $\delta 6.42-7.70$ which were indicative of $1 H$-quinolin-2-one derivatives. In the ${ }^{13} \mathrm{C} \mathrm{NMR}$ spectra, signals in the region $\delta$ 107-156 for aromatic carbons, $\delta 161-163$ for $\mathrm{C}=\mathrm{O}, \delta 155$ for the $\mathrm{C}=\mathrm{N}$ and $\delta 112-151$ for $\mathrm{C}=\mathrm{C}$ of 
the heterocyclic ring as well as signals in the region $\delta 25$ for the $-\mathrm{CH}_{3}$ carbons supported the formation of $\mathbf{4 a - 1}$.

The possible mechanism for the formation of products $\mathbf{4 a - 1}$ is illustrated in Scheme 2. The intermediate I resulting from the initial condensation of the isatin $\mathbf{3}$ with hydrazine hydrate $\mathbf{2}$, followed by nucleophilic substitution on the lactone moiety of the coumarin resulted in the final product 4a-l. This nucleophilic substitution was catalyzed by $\mathrm{InCl}_{3}$ which enhances the electrophilicity of the carbonyl group of the lactone moiety of the coumarin. The nucleophilic substitution was followed by dehydration under the reaction conditions.

\section{Conclusions}

In conclusion, we have developed an unprecedented, original three-component, one-pot approach for the synthesis of highly substituted $1 H$-quinolin-2-one derivatives under microwave irradiation using $\mathrm{InCl}_{3}$ as a catalyst. The experimental simplicity, high yield of products, recyclability of the $\mathrm{InCl}_{3}$ catalyst, and the short reaction times associated with the method presented here for the synthesis of the hitherto unknown $1 \mathrm{H}$-quinolin-2-one derivatives renders it to be of broad interest for synthetic and medicinal chemistry and can be applied for the synthesis of other related compounds.

\section{Experimental Section}

General. All chemicals used were of reagent grade and used as received without further purification. Melting points were determined in an open glass capillary method and are uncorrected. A laboratory microwave oven BP-310 was used for the syntheses. ${ }^{1} \mathrm{H}-\mathrm{NMR}$ spectra were recorded at $400 \mathrm{MHz}$ and ${ }^{13} \mathrm{C}-\mathrm{NMR}$ Spectra at $100 \mathrm{MHz}$ on a Bruker Avance DPX FT spectrometer in $\mathrm{CDCl}_{3}$ using TMS as an internal reference. Mass spectra were determined on a JEOL SX-102 (FAB) mass spectrometer at $70 \mathrm{eV}$. Elemental analysis was carried out using a Coleman automatic $\mathrm{C}, \mathrm{H}$ and $\mathrm{N}$ analyser. The progress of the reaction was monitored by TLC (Merk silica gel).

\section{Typical procedure for the synthesis of $1 \mathrm{H}$-quinolin-2-ones}

A mixture of 4-methyl-coumarin 1 (4 mmol), hydrazine hydrate 2 (6 mmol), isatin 3 (4 mmol) and $10 \mathrm{~mol} \%$ of $\mathrm{InCl}_{3}$ were added to ethanol $(20 \mathrm{ml})$. The reaction mixture was stirred well for 5 min. After that the reaction mixture was subjected to microwave irradiation at $60{ }^{\circ} \mathrm{C}$ for the time given in the Table 1 and the progress of the reaction was monitored by TLC. After the completion of the reaction, the reaction mixture is cooled to room temperature and washed with cold ethanol. The product was dried and purified by column chromatography (ethyl acetate: $n$ hexane 2:8). 
7-Hydroxy-4-methyl-1-(2-oxo-1,2-dihydro-indol-3-ylideneamino)-1H-quinolin-2-one (4a).

${ }^{1} \mathrm{H}-\mathrm{NMR}: \delta=1.71\left(\mathrm{~s}, 3 \mathrm{H}, \mathrm{CH}_{3}\right), 5.0\left(\mathrm{~s}, 1 \mathrm{H}, \mathrm{OH}\right.$, exchangeable with $\left.\mathrm{D}_{2} \mathrm{O}\right), 6.35(\mathrm{~s}, 1 \mathrm{H}, \mathrm{CH})$, 6.42-7.70 (m, 7H, Ar-H), 8 (s, 1H, NH). ${ }^{13} \mathrm{C}-\mathrm{NMR}: \delta=25.1,107.5,111.2,112.4,119.4,120.5$, 123.1, 124.0, 124.2, 127.8, 129.2, 131.0, 137.3, 138.7, 151.0, 155, 156.7, 161, 163. MS (EI): $\mathrm{m} / \mathrm{z}$ $319\left(\mathrm{M}^{+}\right)$. Anal. Calcd. For $\mathrm{C}_{18} \mathrm{H}_{13} \mathrm{~N}_{3} \mathrm{O}_{3}$ (319): C, 67.71; H, 4.10; N, 13.16\%. Found: C, 67.70; $\mathrm{H}, 4.10 ; \mathrm{N}, 13.25 \%$.

6-Hydroxy-4-methyl-1-(2-oxo-1,2-dihydro-indol-3-ylideneamino)-1H-quinolin-2-one (4b). ${ }^{1} \mathrm{H}-\mathrm{NMR}: \delta=1.71\left(\mathrm{~s}, 3 \mathrm{H}, \mathrm{CH}_{3}\right), 5.0\left(\mathrm{~s}, 1 \mathrm{H}, \mathrm{OH}\right.$, exchangeable with $\left.\mathrm{D}_{2} \mathrm{O}\right), 6.35(\mathrm{~s}, 1 \mathrm{H}, \mathrm{CH}), 6.59-$ $7.70(\mathrm{~m}, 7 \mathrm{H}, \mathrm{Ar}-\mathrm{H}), 8.0(\mathrm{~s}, 1 \mathrm{H}, \mathrm{NH}) .{ }^{13} \mathrm{C}-\mathrm{NMR}: \delta=25.1,112.4,113.6,115.1,120.5,121.7$, $123.1,124.2,128.2,128.5,129.2,131.0,138.7,151.0,152.8,155.0,161.0,163.0 . \mathrm{MS}(\mathrm{EI}): \mathrm{m} / z$ $319\left(\mathrm{M}^{+}\right)$. Anal. Calcd. For $\mathrm{C}_{18} \mathrm{H}_{13} \mathrm{~N}_{3} \mathrm{O}_{3}$ : C, 67.71; H, 4.10; N, $13.16 \%$. Found: C, 67.78; H, $4.21 ; \mathrm{N}, 13.11 \%$.

5,7-Dihydroxy-4-methyl-1-(2-oxo-1,2-dihydro-indol-3-ylideneamino)-1H-quinolin-2-one (4c). ${ }^{1} \mathrm{H}-\mathrm{NMR}: \delta=1.71\left(\mathrm{~s}, 3 \mathrm{H}, \mathrm{CH}_{3}\right), 5.0\left(\mathrm{~s}, 2 \mathrm{H}, 2 \mathrm{xOH}\right.$, exchangeable with $\left.\mathrm{D}_{2} \mathrm{O}\right), 6.35(\mathrm{~s}, 1 \mathrm{H}, \mathrm{CH})$, 5.89-7.70 (m, 6H, Ar-H), $8.0(\mathrm{~s}, 1 \mathrm{H}, \mathrm{NH}) .{ }^{13} \mathrm{C}-\mathrm{NMR}: \delta=25.4,98.4,100.1,106.6,112.4,120.5$, 123.1, 124.2, 129.2, 131.0, 138.7, 151.0, 155.0, 156.7, 158.1, 161.0, 163.0. MS (EI): $\mathrm{m} / z 335$ $\left(\mathrm{M}^{+}\right)$. Anal. Calcd. For $\mathrm{C}_{18} \mathrm{H}_{13} \mathrm{~N}_{3} \mathrm{O}_{4}: \mathrm{C}, 64.47 ; \mathrm{H}, 3.91 ; \mathrm{N}, 12.53 \%$. Found: C, 64.40; H, 3.98; N, $12.61 \%$.

7-Methoxy-4-methyl-1-(2-oxo-1,2-dihydro-indol-3-ylideneamino)-1H-quinolin-2-one (4d). ${ }^{1} \mathrm{H}-\mathrm{NMR}: \delta=1.71\left(\mathrm{~s}, 3 \mathrm{H}, \mathrm{CH}_{3}\right), 3.73\left(\mathrm{~s}, 3 \mathrm{H}, \mathrm{OCH}_{3}\right), 6.35$ (s, 1H, CH), 6.46-7.70 (m, 7H, Ar-H), $8.0(\mathrm{~s}, 1 \mathrm{H}, \mathrm{NH}) .{ }^{13} \mathrm{C}-\mathrm{NMR}: \delta=25.1,56.0,105.9,109.6,112.4,119.1,120.5,123.1,124.2,127.4$, 129.2, 131.0, 136.9, 138.7, 151.0, 155.0, 161.0, 161.4, 163.0. MS (EI): $m / z 333\left(\mathrm{M}^{+}\right)$. Anal. Calcd. For $\mathrm{C}_{19} \mathrm{H}_{15} \mathrm{~N}_{3} \mathrm{O}_{3}$ : C, 68.46; H, 4.54; N, 12.61\%. Found: C, 68.52; H, 4.51; N, $12.68 \%$.

7-Hydroxy-4-methyl-1-(5-nitro-2-oxo-1,2-dihydro-indol-3-ylideneamino)-1H-quinolin-2-one (4e). ${ }^{1} \mathrm{H}-\mathrm{NMR}: \delta=1.71\left(\mathrm{~s}, 3 \mathrm{H}, \mathrm{CH}_{3}\right), 5.0\left(\mathrm{~s}, 1 \mathrm{H}, \mathrm{OH}\right.$, exchangeable with $\left.\mathrm{D}_{2} \mathrm{O}\right), 6.35(\mathrm{~s}, 1 \mathrm{H}, \mathrm{CH})$, 6.42-8.5 (m, 6H, Ar-H), $8.0(\mathrm{~s}, 1 \mathrm{H}, \mathrm{NH}) .{ }^{13} \mathrm{C}-\mathrm{NMR}: \delta=25.1,107.5,111.2,112.4,119.4,121.4$, 124.0, 124.3, 126.1, 127.8, 137.3, 144.1, 144.8, 151.0, 155.0, 156.7, 161.0, 163.0. MS (EI): $\mathrm{m} / z$ $364\left(\mathrm{M}^{+}\right)$. Anal. Calcd. For $\mathrm{C}_{18} \mathrm{H}_{12} \mathrm{~N}_{4} \mathrm{O}_{5}$ : C, 59.34; H, 3.32; N, 15.38\%. Found: C, 59.26; H, 3.39; $\mathrm{N}, 15.41 \%$.

6-Hydroxy-4-methyl-1-(5-nitro-2-oxo-1,2-dihydro-indol-3-ylideneamino)-1H-quinolin-2-one (4f). ${ }^{1} \mathrm{H}-\mathrm{NMR}: \delta=1.71\left(\mathrm{~s}, 3 \mathrm{H}, \mathrm{CH}_{3}\right), 5.0\left(\mathrm{~s}, 1 \mathrm{H}, \mathrm{OH}\right.$, exchangeable with $\left.\mathrm{D}_{2} \mathrm{O}\right), 6.35(\mathrm{~s}, 1 \mathrm{H}, \mathrm{CH})$, 6.59-8.5 (m, 6H, Ar-H), $8.0(\mathrm{~s}, 1 \mathrm{H}, \mathrm{NH}) .{ }^{13} \mathrm{C}-\mathrm{NMR}: \delta=25.1,112.4,113.6,115.1,121.4,121.7$, 124.0, 124.3, 126.1, 128.2, 144.1, 144.8, 151.0, 152.8, 155.0, 161.0, 163.0. MS (EI): $m / z 364$ $\left(\mathrm{M}^{+}\right)$. Anal. Calcd. For $\mathrm{C}_{18} \mathrm{H}_{12} \mathrm{~N}_{4} \mathrm{O}_{5}$ : C, 59.34; H, 3.32; N, 15.38\%. Found: C, 59.42; H, 3.37; N, $15.41 \%$.

5,7-Dihydroxy-4-methyl-1-(5-nitro-2-oxo-1,2-dihydro-indol-3-ylideneamino)-1H-quinolin-2one (4g). ${ }^{1} \mathrm{H}-\mathrm{NMR}: \delta=1.71\left(\mathrm{~s}, 3 \mathrm{H}, \mathrm{CH}_{3}\right), 5.0\left(\mathrm{~s}, 2 \mathrm{H}, 2 \mathrm{xOH}\right.$, exchangeable with $\left.\mathrm{D}_{2} \mathrm{O}\right), 6.35$ (s, $1 \mathrm{H}, \mathrm{CH}), 5.89-8.5(\mathrm{~m}, 5 \mathrm{H}, \mathrm{Ar}-\mathrm{H}), 8.0(\mathrm{~s}, 1 \mathrm{H}, \mathrm{NH}) .{ }^{13} \mathrm{C}-\mathrm{NMR}: \delta=25.4,98.4,100.1,106.6$, $112.4,121.4,124.0,124.3,126.1,138.7,144.1,144.8,151.0,155.0,156.6,161.0,163.0 . \mathrm{MS}$ 
(EI): $m / z, 380\left(\mathrm{M}^{+}\right)$. Anal. Calcd. For $\mathrm{C}_{18} \mathrm{H}_{12} \mathrm{~N}_{4} \mathrm{O}_{6}$ : C, 56.85; H, 3.18; N, 14.73 \%. Found: C, $56.93 ; \mathrm{H}, 3.20 ; \mathrm{N}, 14.84 \%$.

7-Methoxy-4-methyl-1-(5-nitro-2-oxo-1,2-dihydro-indol-3-ylideneamino)-1H-quinolin-2-one (4h). ${ }^{1} \mathrm{H}-\mathrm{NMR}: \delta=1.71\left(\mathrm{~s}, 3 \mathrm{H}, \mathrm{CH}_{3}\right.$ ), 3.73 (s, 3H, $\left.\mathrm{OCH}_{3}\right), 6.35$ (s, 1H, CH), 6.46-8.5 (m, 6H, Ar-H), $8.0(\mathrm{~s}, 1 \mathrm{H}, \mathrm{NH}) .{ }^{13} \mathrm{C}-\mathrm{NMR}: \delta=25.4,56.0,105.9,109.6,112.4,119.1,121.4,124.0$, 124.3, 126.1, 127.4, 136.9, 144.1, 144.8, 151.0, 155.0, 156.6, 161.0, 161.4, 163.0. MS (EI): $\mathrm{m} / z$ $378\left(\mathrm{M}^{+}\right)$. Anal. Calcd. For $\mathrm{C}_{19} \mathrm{H}_{14} \mathrm{~N}_{4} \mathrm{O}_{5}$ : C, 60.32; H, 3.73; N, 14.81\%. Found: C, 60.29; H, 3.65; $\mathrm{N}, 14.89 \%$.

7-Hydroxy-4-methyl-1-(5-methyl-2-oxo-1,2-dihydro-indol-3-ylideneamino)-1H-quinolin-2-one (4i). ${ }^{1} \mathrm{H}-\mathrm{NMR}: \delta=1.71\left(\mathrm{~s}, 3 \mathrm{H}, \mathrm{CH}_{3}\right), 2.35$ (s, 3H, $\left.\mathrm{OCH}_{3}\right), 5.0$ (s, 1H, OH, exchangeable with $\left.\mathrm{D}_{2} \mathrm{O}\right), 6.35(\mathrm{~s}, 1 \mathrm{H}, \mathrm{CH}), 6.42-7.6(\mathrm{~m}, 6 \mathrm{H}, \mathrm{Ar}-\mathrm{H}), 8.0(\mathrm{~s}, 1 \mathrm{H}, \mathrm{NH}) .{ }^{13} \mathrm{C}-\mathrm{NMR}: \delta=20.9,25.1$, 107.5, 111.2, 112.4, 119.1, 120.4, 123.0, 127.8, 129.9, 131.7, 133.4, 135.7, 147.3, 151.0, 155.0, 156.7, 161.0, 163.0. MS (EI): $m / z 333\left(\mathrm{M}^{+}\right)$. Anal. Calcd. For $\mathrm{C}_{19} \mathrm{H}_{15} \mathrm{~N}_{3} \mathrm{O}_{3}: \mathrm{C}, 68.46 ; \mathrm{H}, 4.54 ; \mathrm{N}$, $12.61 \%$. Found: C, 68.43; H, 4.61; N, $12.56 \%$.

6-Hydroxy-4-methyl-1-(5-methyl-2-oxo-1,2-dihydro-indol-3-ylideneamino)-1H-quinolin-2-one (4j). ${ }^{1} \mathrm{H}-\mathrm{NMR}: \delta=1.71\left(\mathrm{~s}, 3 \mathrm{H}, \mathrm{CH}_{3}\right), 2.35$ (s, 3H, $\left.\mathrm{OCH}_{3}\right), 5.0$ (s, 1H, OH, exchangeable with $\left.\mathrm{D}_{2} \mathrm{O}\right), 6.35(\mathrm{~s}, 1 \mathrm{H}, \mathrm{CH}), 6.59-7.6(\mathrm{~m}, 6 \mathrm{H}, \mathrm{Ar}-\mathrm{H}), 8.0(\mathrm{~s}, 1 \mathrm{H}, \mathrm{NH}) .{ }^{13} \mathrm{C}-\mathrm{NMR}: \delta=20.9,25.1$, 112.4, 113.6, 115.1, 120.4, 121.7, 123.0, 128.2, 128.5, 129.9, 131.7, 133.4, 135.7, 151.0, 152.8, 155.0, 161.0, 163.0. MS (EI): $m / z 333\left(\mathrm{M}^{+}\right)$. Anal. Calcd. For $\mathrm{C}_{19} \mathrm{H}_{15} \mathrm{~N}_{3} \mathrm{O}_{3}: \mathrm{C}, 68.46 ; \mathrm{H}, 4.54 ; \mathrm{N}$, $12.61 \%$. Found: C, 68.49; H, 4.61; N, $12.68 \%$.

5,7-Dihydroxy-4-methyl-1-(5-methyl-2-oxo-1,2-dihydro-indol-3-ylideneamino)-1H-quinolin -2-one (4k). ${ }^{1} \mathrm{H}-\mathrm{NMR}: \delta=1.71\left(\mathrm{~s}, 3 \mathrm{H}, \mathrm{CH}_{3}\right), 2.35\left(\mathrm{~s}, 3 \mathrm{H}, \mathrm{OCH}_{3}\right), 5.0(\mathrm{~s}, 2 \mathrm{H}, 2$ x $\mathrm{OH}$, exchangeable with $\left.\mathrm{D}_{2} \mathrm{O}\right), 6.35(\mathrm{~s}, 1 \mathrm{H}, \mathrm{CH}), 5.89-7.6(\mathrm{~m}, 5 \mathrm{H}, \mathrm{Ar}-\mathrm{H}), 8.0(\mathrm{~s}, 1 \mathrm{H}, \mathrm{NH}) .{ }^{13} \mathrm{C}-\mathrm{NMR}$ : $\delta=20.9,25.4,98.4,100.1,106.6,112.4,120.4,123.0,129.9,131.7,133.4,135.7,138.7,151.0$, 155.0, 156.6, 158.1, 161.0, 163.0. MS (EI): $\mathrm{m} / \mathrm{z} 349\left(\mathrm{M}^{+}\right)$. Anal. Calcd. For $\mathrm{C}_{19} \mathrm{H}_{15} \mathrm{~N}_{3} \mathrm{O}_{4}$ : C, 65.32; H, 4.33; N, $12.03 \%$. Found: C, 65.40; H, 4.41; N, $12.08 \%$.

7-Methoxy-4-methyl-1-(5-methyl-2-oxo-1,2-dihydro-indol-3-ylideneamino)-1H-quinolin-2one (4l). ${ }^{1} \mathrm{H}-\mathrm{NMR}: \delta=1.71\left(\mathrm{~s}, 3 \mathrm{H}, \mathrm{CH}_{3}\right), 2.35\left(\mathrm{~s}, 3 \mathrm{H}, \mathrm{OCH}_{3}\right), 3.73(\mathrm{~s}, 3 \mathrm{H}, \mathrm{OH}$, exchangeable with $\left.\mathrm{D}_{2} \mathrm{O}\right), 6.35$ (s, $\left.1 \mathrm{H}, \mathrm{CH}\right), 6.46-7.6(\mathrm{~m}, 6 \mathrm{H}, \mathrm{Ar}-\mathrm{H}), 8.0$ (s, $\left.1 \mathrm{H}, \mathrm{NH}\right) .{ }^{13} \mathrm{C}-\mathrm{NMR}: \delta=20.9,25.1$, 56.0, 105.9, 109.6, 112.4, 119.1, 120.4, 123.0, 127.4, 129.9, 131.7, 133.4, 135.7, 136.9, 151.0, 155.0, 161.0, 161.4, 163.0. MS (EI): $m / z, 347\left(\mathrm{M}^{+}\right)$. Anal. Calcd. For $\mathrm{C}_{20} \mathrm{H}_{17} \mathrm{~N}_{3} \mathrm{O}_{3}$ : C, 69.15; $\mathrm{H}$, 4.93; N, 12.10\%. Found: C, 69.13; H, 4.89; N, 12.15\%.

\section{Acknowledgements}

We gratefully acknowledge financial support from CSIR, New Delhi, India. Authors are also thankful to RSIC, CDRI, Lucknow and IISc Bangalore India, for providing elemental and spectral analysis. 


\section{References}

1. Doube, D.; Blouin, M.; Brideau, C.; Chan, C.; Desmarais, C.; Ethier, D.; Falgueyret, J. P.; Friesen, R. W.; Girard, M.; Girard, Y.; Guay, J.; Tagari, P.; Young, R. N. Bioorg. Med. Chem. Lett. 1998, 8, 1255.

2. Kidwai, M.; Bhushan, K. R.; Sapra, P.; Saxena, R. K.; Gupta, R. Bioorg. Med. Chem. 2000, $8,69$.

3. Moissev, I. K.; Zemtsova, M. N.; Trakhtenberg, P. L.; Kulikowa, D. A.; Pskobkina, I.; Neshchadim, G. N.; Ostapchuk, N. V. Khim. Farm. Zh. 1998, 22, 1448.

4. Craig, J. C.; Person, P. E. J. Med. Chem. 1971, 14, 1221.

5. Narsinh, D.; Anamik, S. Ind. J. Pharm. Sci. 2001, 63, 211.

6. Dillard, R. D.; Pavey, D. E.; Benslay, D. N. J. Med. Chem. 1973, 16, 251.

7. (a) Aggarwal A.K. and Jenekhe S. A., Macromolecules 1991, 24, 6806. (b) Zhang X., Shetty A. S. and Jenekhe S. A. Macromolecules 1999, 32, 7422. (c) Jenekhe S. A., Lu L. and Alam M. M., Macromolecules 2001, 34, 7315.

8. Polanski, J.; Niedbala, H.; Musiol, R.; Podeszwa, B.; Tabak, D.; Palka, A.; Mencel, A.; Finster, J.; Mouscadet, J. F.; Le Bret, M. Lett. Drugs Des. Disc. 2006, 3, 175.

9. Polanski, J.; Niedbala, H.; Musiol, R.; Podeszwa, B.; Tabak, D.; Palka, A.; Mencel, A.; Mouscadet, J. F.; Le Bret, M. Lett. Drugs Des. Disc. 2007, 4, 99.

10. Teitz, Y.; Barko, N.; Abramoff, M.; Ronen, D. Chemotherapy 1994, 40, 195.

11. Ronen, D.; Teitz, Y. Antimicrob. Agents Chemother. 1984, 26, 913.

12. Teitz, Y.; Ronen, D.; Vansover, A.; Stematsky, T.; Riggs, J. L. Antiviral Res. 1994, 24, 305.

13. Sherman, L.; Edelstein, F.; Shtacher, G.; Avramoff, M.; Teitz, Y. J. Gen. Virol. 1980, 46, 195.

14. Teitz Y.; Ronen D.; Vnsover A.; Stematsky T.; Rigg J. L. Antiviral. Res. 1994; 24; 305.

15. Pauwels R.; Balzarini J.; Baba M.; Snoeck R.; Schols D. J.; Herdewijin P. ; Desmyter J.; Clercq E. D. J. Virol. Methods 1988; 20; 309.

16. (a) Loh, T.-P.; Pei, J. J. Chem. Soc., Chem. Commun. 1996, 2315. (b) Babu, G.; Perumal, P. T. Aidrichim. Acta 2000, 33, 16. (c) Ranu, B. C. Eur. J. Org. Chem. 2000, 2347. (d) Li, J.; Li, C. J. Tetrahedron Lett. 2001, 42, 793.

17. Ranu, B. C. Eur J. Org. Chem. 2000, 2347

18. Paquette, L. A; Mitzal, J. M. J. Am. Chem. Soc. 1996, 118, 1937

19. (a) Zhu, J.; Bienayme, H. Multicomponent Reactions; Wiley-VCH: Weinheim, 2005. (b) Dömling, A.; Ugi, I. Angew. Chem., Int. Ed. 2000, 39, 3168. (c) Ganem, B. Acc. Chem. Res. 2009, 42, 463.

20. (a) McGee, D. P. C.; Martin, J. C.; Smee, D. F.; Verheyden, J. P. H. Nucleosides and Nucleotides 1990, 9, 815. (b) Ugi, I.; Domling, A. Endeavour 1994, 18, 115. (c) Kraus, G. A.; Nagy, J. O. Tetrahedron 1985, 41, 3537. 
21. (a) Zeigler, T.; Kaisers, H. J.; Schlomer, R.; Koch, C. Tetrahedron 1999, 55, 8397. (b) Caddick, S. Tetrahedron 1995, 51, 10403. (c) Meshram, H. M.; Sekhar, K. C.; Ganesh, Y. S. S.; Yadav, J. S. Synlett 2000, 1273.

22. (a) Al-Bayati, R. I.; Al-Amiery, A. A. H.; Al-Majedy, Y. K. Afr. J. Pure Appl. Chem. 2010, 4(6), 74. (b) Bishnoi, A.; Saxena, R. Indian J. Heterocyclic Chem. 2001, 11, 4750. 